\title{
Chronic Fatigue Syndrome and Bone Marrow Defects of the Jaw - A Case Report on Additional Dental X-Ray Diagnostics with Ultrasound
}

\author{
Johann Lechner (D) \\ Fabian Schick \\ Clinic for Integrative Dentistry, Munich, \\ Germany
}

Purpose: This paper aims to demonstrate the additional benefit of ultrasound in the diagnosis of chronic osteolysis and osteonecrosis (bone marrow defects) of the jaw shown in a clinical case report.

Patients and Methods: A case of chronic fatigue syndrome (CFS) in a young man presenting the typical, ambiguous symptoms, which were accompanied by headaches and tinnitus. X-ray techniques, namely panoramic radiographs (OPG) and cone beam computed tomography (DVT/ $\mathrm{CBCT}$ ), failed to produce any remarkable findings of bone marrow defects (BMDJ) in the jawbone. However, the measurement of bone density using trans-alveolar ultrasound (TAU) indicated a possible bone marrow defect in the lower left jawbone.

Results: Surgery was undertaken at the conspicuous area. Additional to softened, ischemic, fatty tissue, a black area was revealed, which was surprisingly subsequently identified as aspergillosis by histopathological analysis. In addition, the excessive local RANTES/CCL5 expression found in the affected area confirmed the necessity for surgical debridement and additional findings of TAU.

Conclusion: In contrast to radiography, complementary TAU imaging of the BMDJ revealed chronic inflammatory signaling RANTES/CCL5 pathways and fungal colonization. This case report supports the need for additional diagnostic techniques beyond radiographic modalities, which can help to elucidate the diagnostic composition and knowledge of the bone manifestations of systemic diseases.

Keywords: chronic fatigue syndrome, radiographic diagnostics, bone marrow defect, osteonecrosis, RANTES/CCL5, trans-alveolar ultrasonography

\section{Introduction}

With the increasing complexity of various systemic diseases, it is necessary to determine their underlying pathogenesis. Autonomic dysfunction with poorly defined multisystem disorders frequently presents a clinical challenge for medical practitioners. ${ }^{1,2}$ In the absence of an identifiable cause, and given the insufficient research available on this increasingly common phenomenon, presentations of "idiopathic multimorbidity" are often assumed to be of psychogenic origin, and pharmacological interventions such as psychoactive medications are most commonly prescribed. ${ }^{3,4}$ A case report from our clinic supports the need for expanded diagnostic techniques, which can help elucidate the pathogenesis associated with systemic dysregulation. A common dysregulation today is the Chronic Fatigue Syndrome, as in the case report presented here. 


\section{Clinical Case}

Here we describe a case of Chronic Fatigue Syndrome (CFS) in a young man presenting with typical, ambiguous symptoms that are characteristic of this syndrome and which were accompanied by headaches and tinnitus. Given the unclear etiology of this patient's presentation, we associated this case in our clinic with avascular, aseptic-osteolytic, and inflammatory bone marrow defects (BMDJ) in the jawbone, collectively referred to as "silent inflammation". 5 The patient, 28 years of age, had been suffering from CFS for approximately four years. His symptoms included dizziness, the inability to work, impaired concentration, and depression. Previous treatment approaches, which were primarily psychologically oriented, showed no significant improvement. As a last resort, it was recommended that interference fields caused due to teeth and jawbone be investigated. The patient thus sought treatment at our clinic. The patient provided his written informed consent to participate in this report and to have the case details and any accompanying images published. Institutional approval was not required to publish the case details. The patient was given clear information that not all additional examination methods and surgical techniques have been generally accepted scientifically to date.

\section{Clinical Investigations and Findings}

The patient received the expert diagnosis of CFS after immunological and serological examinations and accompanying psychological counseling. In addition to taking the patient's full medical history and bimanual palpation of the bilateral lymph nodes, the radiographic exam was performed to determine whether previously undetected inflammation was present. In addition to the radiographs, a newly developed trans-alveolar ultrasound (TAU) device was used.

\section{Radiographic Findings with 2D-OPG and DVT/CBCT}

A two-dimensional orthopantomogram (OPG) and digital volume tomogram (DVT) combined with cone beam computed tomography (CBCT) were performed to provide a panoramic image of the patient's mouth (DVT/CBCT). Conventional OPG failed to show any abnormal findings in the wisdom tooth and retromolar region at area 38/39 (left panel, Figure 1). With the use of DVT/CBCT, it is possible to depict the medullary structures in the area of a bone marrow defect in the jawbone (BMDJ) with greater reliability. ${ }^{6}$ However, the sectional image of the patient's jaw obtained using DVT/CBCT (right panel, Figure 1) also failed to show any further resolution of the bony trabecular structures at area 38and retromolar area. Based on these radiographic findings, an inflammatory process in the jaw area would be ruled out, and, accordingly, maxillofacial surgery determined not to be required. Currently, magnetic resonance imaging (MRI) is the method of choice for assessing osteonecrosis, which was not available in this case.

\section{Trans-Alveolar Ultrasound Bone Density Measurement}

In an earlier publication, ${ }^{7}$ we demonstrated the fundamental problem associated with the use of diagnostic radiography when attempting to identify osteonecrosis and inflammatory osteolysis in cancellous bone. To supplement potentially unreliable X-ray findings, we used a radiation-free method known as trans-alveolar ultrasound (TAU) measurement, which determines the bone density in the jaw. ${ }^{8}$ TAU devices in dentistry and their usefulness to measure bone density were described earlier in the scientific literature. ${ }^{9,10}$ TAU measurements are based on ultrasonic principles where sound is best conducted through solid material, more weakly in aqueous environments, and slowest through the air. When the measurement is obtained, the ultrasonic pulse is attenuated; solid structures are able to attenuate sound to a weaker degree than fatty or aqueous structures. In order to indicate both physical and structural changes, the TAU device creates digital (twoand three-dimensional) images with corresponding color coding (Figure 2). The TAU device consists of an ultrasonic transmitter that is placed on the skin over the specific tooth and jaw area to be measured. A receiver, which is the size of a thumbnail, is also placed intraorally over the specific dental area to be assessed. Interference-free acoustic coupling is achieved with a gel cushion that is placed intraorally and extra orally (Figure 3). Each dental area is measured individually. The receiver has 91 piezoelectric fields that register the trans-alveolar sound waves. These sound waves are converted into a colored pulse via a computer unit whereby sound waves of varying speeds are represented in different colors. The TAU monitor displays the various structures detected based on mineralization density using two- and three-dimensional graphic representations of bone density. ${ }^{9-12}$ 

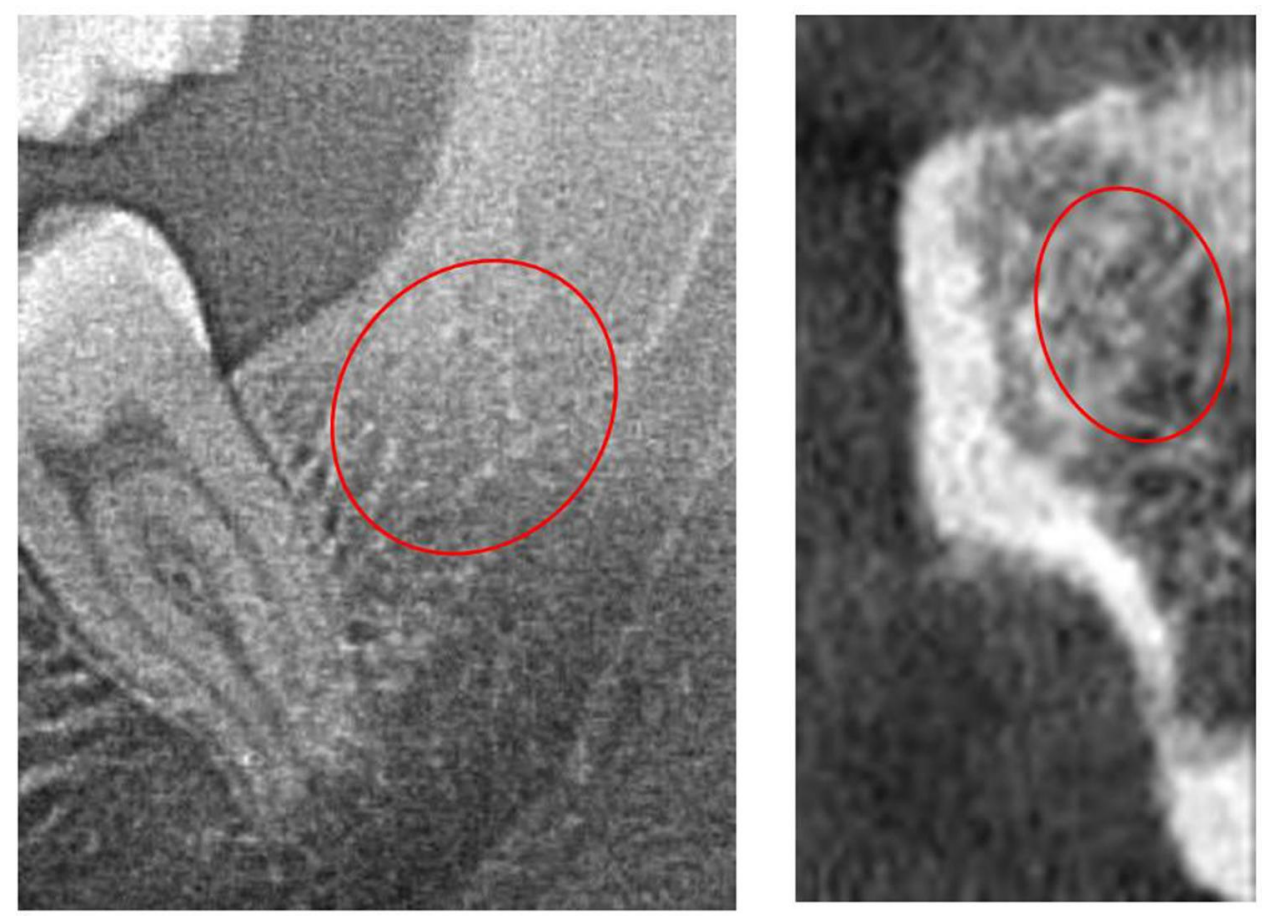

Figure I Panoramic radiograph OPG and sectional DVT/CBCT image.

Notes: Left panel: panoramic 2D-OPG image shows no abnormal findings at area 38 and retromolar area (39). Significance of the red circles: The red circles show the radiographically inconspicuous areas of the bone medulla, where OPG and DVT/CBCT failed to show any abnormal findings in the wisdom tooth and retromolar area. Right panel: sectional 3D-DVT image also shows no resolution of the bony trabecular structures at area 38/39.

Abbreviations: 2D-OPG, two-dimensional orthopantomogram; 3D-DVT, three-dimensional digital volume tomogram.

\section{Surgical Repair of Area 38 and Retromolar Area}

The findings of the TAU measurement performed on the patient indicated that there was a possible bone marrow defect (BMDJ) at area 38. As such, we decided to surgically debride area 38, extending beyond into so-called "area 39". Local anesthesia was administered from areas 37 to retromolar area 39, and, following this, a mucoperiosteal flap was created and folded down. The cortical bone of the jaw at area $38 / 39$ was over-supplied with blood and removed at the retromolar area. With the intraosseous region now visible, the area was curetted (Figure 4), and the findings identified in the medullary cavity were surprising (Figure 5). Plateletrich fibrin was applied to promote tissue healing.

\section{Postoperative Diagnosis Following Oral Surgical Debridement of Left Lower Molar/Retromolar Area Bone Marrow Defect Morphology}

Throughout the entire medullary area spanning from region 38 to retromolar area 39 , spongiose tissue had degenerated into fatty, ischemic tissue and did not indicate any healing tendencies. (Figure 3). This connective tissue was easily scooped out and collected as a sample. The left panel of Figure 5 shows a black mass that had formed along with typical fatty-degenerative, ischemic cancellous bone.

\section{Histological Findings}

The histopathological findings of the excised mass from area $38 / 39$ was described as follows:

Amorphous, nucleus-free substance, which showed fungal hyphae at the margin, and included hyphae that had recently started sprouting. This fungus may be Candida, but early-stage Aspergillus is also possible. Scar tissue with considerable cellular inflammation was present, whereby lymphocytes, plasma cells, and fibroblasts, as well as multinucleated giant cells of the foreign-body type and plenty of foam-cell macrophages, were identified. The extent of chronic inflammation, which was predominant, was quite considerable. An apparently devitalized bone fragment, with adipose tissue originating from the bone marrow cavity, also demonstrates chronic inactive inflammation, as well as colonization with small fungal spores. 


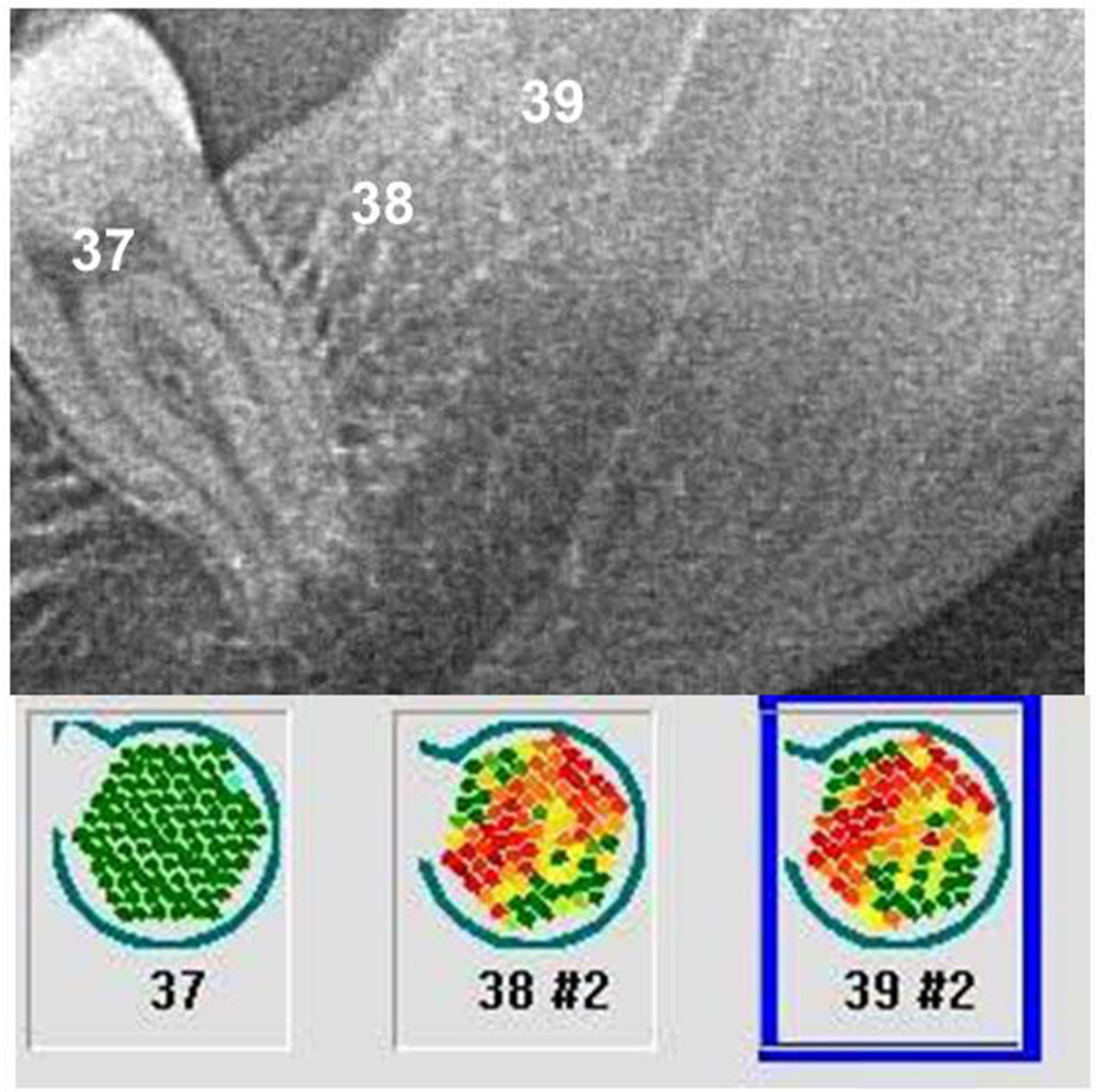

Figure 2 Comparison of panoramic radiograph (OPG) and TAU measurement which shows specific via green dense, inconspicuous structures) and red (low-density, conspicuous structures) resolution.

Notes: In contrast to the panoramic radiograph and DVT/CBCT images, the TAU measurements with respect to the edentulous retromolar area $38 / 39$ clearly indicated the suspicion of osteolysis of the jaw This finding was a result of increased attenuation of the ultrasound beam due to fatty degeneration of the dense medullary cancellous bone. This may be compared to the finding for the area at healthy tooth 37 which showed a solid bone structure and bone density in green.

Abbreviations: OPG, two-dimensional panoramic radiograph; TAU, trans-alveolar ultrasound; DVT, three-dimensional digital volume tomogram; CBCT, cone beam computed tomography.

\section{Local Hyperactivation of Immune Mediator Chemokine RANTES/CCL5 in the Osteolytic Jawbone}

In the fatty tissue samples obtained from the softened region of the patient's jaw at area 38 and retromolar area 39, the multiplex analysis of the laboratory showed $6218 \mathrm{pg} / \mathrm{mL}$ of the proinflammatory chemokine RANTES/CCL5 (R/C), representing a nearly 40 -fold overexpression; the normal finding for $\mathrm{R} / \mathrm{C}$ in healthy medullary maxillary spongial bone is 149.9 $\mathrm{pg} / \mathrm{mL}$ (average age, 51.4 years; range, 33-72 years; gender (female/male): 10/9) (Figure 6). ${ }^{13,14}$ In a previous case report, we demonstrated the immunological connection between the local overexpression of $\mathrm{R} / \mathrm{C}$ found in fatty degenerative osteonecrosis of the jawbone (FDOJ) and systemic disease. ${ }^{15}$

\section{Discussion}

\section{Panoramic Radiograph and DVT/CBCT}

Previous radiographs of the jaw failed to yield any remarkable findings in the patient (Figure 1). This is in coincidence with our hypothesis that the most common dental diagnostic tool, the panoramic radiograph OPG, is unsuitable for detecting BMDJ/FDOJ. This problem of radiographic imaging contributes to the widespread misjudgment of BMDJ/FDOJ as pathological and - via chronic local R/C overexpression - pathogenetic structural changes of the jawbone. ${ }^{6,11}$ The DVT/CBCT performed in this case also failed to indicate the presence of intraosseous pathology (Figure 1). 


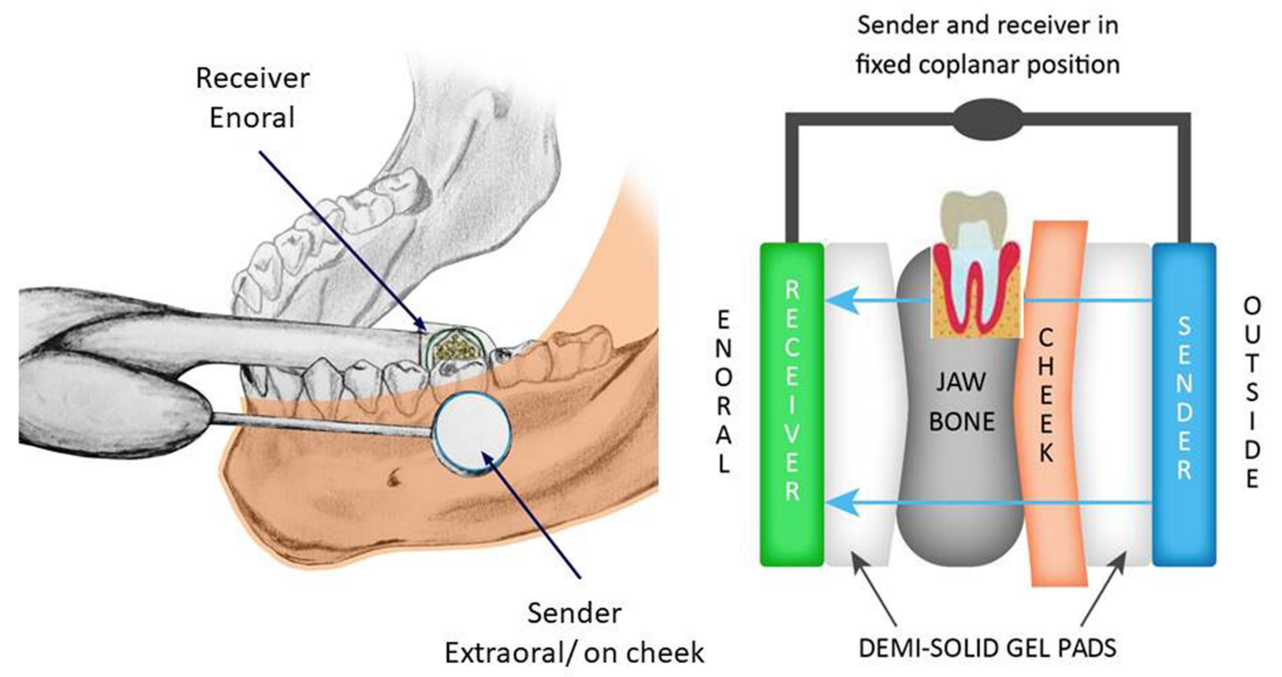

Figure 3 TAU measurement of jawbone density using a TAU device.

Note: Individual adjustments of the measuring unit are made in response to different jaw conditions by means of specially designed gel cushions. " Abbreviation: TAU, trans-alveolar ultrasound.

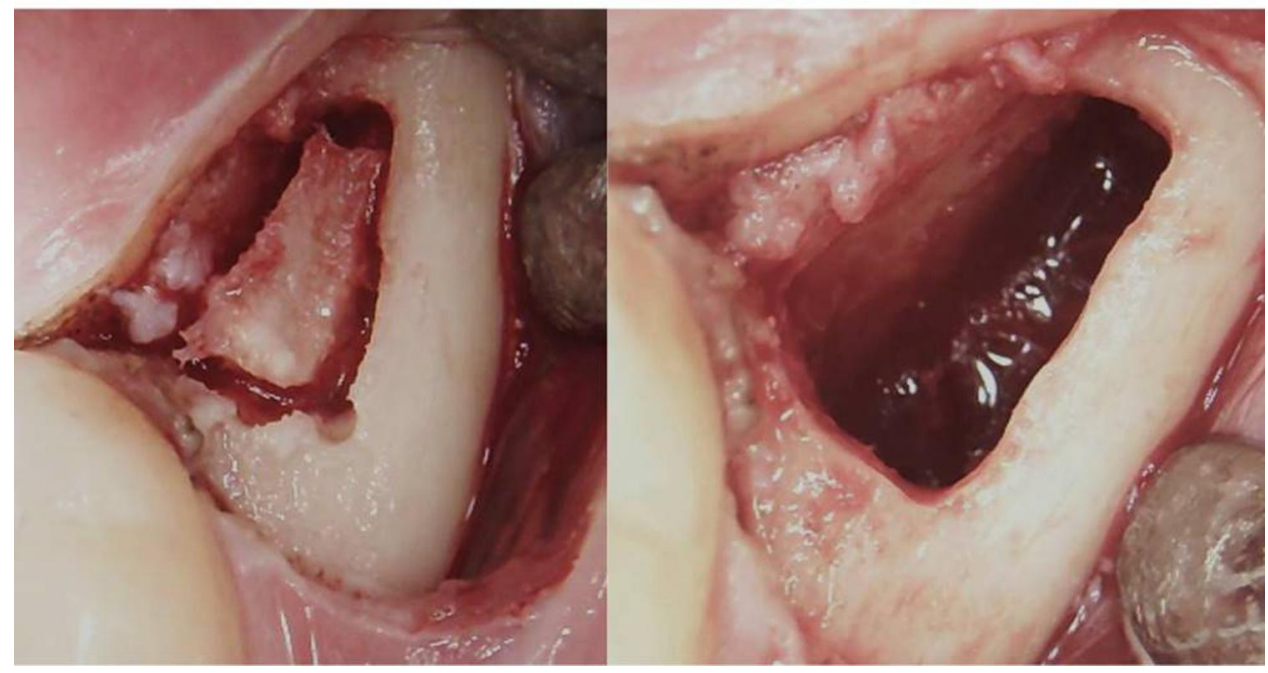

Figure 4 Surgical procedure for a retromolar BMDJ/FDOJ.

Notes: Left panel: after folding down the mucoperiosteal flap, a bone window was formed in the cortex. Right panel: curetted medullary cavity. Abbreviations: BMDJ, bone marrow defect in jawbone; FDOJ, fatty degenerative osteonecrosis of the jawbone.

\section{TAU Measurement of Bone Density}

In contrast to the inconspicuous X-ray images of the patient's jaw, the TAU measurements showed extensive areas of softened and necrotic cancellous bone, often referred to as "cavitations." As in this case, cavitations may often be completely asymptomatic. In our efforts to close the diagnostic gap due to the limitations associated with standard X-ray methods, the application of TAU may be considered a major step forward in the detection of BMDJ/FDOJ.

\section{RANTES/CCL5}

With the analysis of RANTES expression in morphologically and histologically conspicuous jawbone samples, we are entering new territory. ${ }^{13-15}$ Chemokine RANTES/ CCL5 (R/C) is a recognized inflammatory agent in medical research. The progression of chronic inflammatory diseases is often hidden beneath the surface as a result of an immune system that is chronically activated by excess cytokines. These chemokines also stimulate other signaling pathways, the expression of which we have identified 


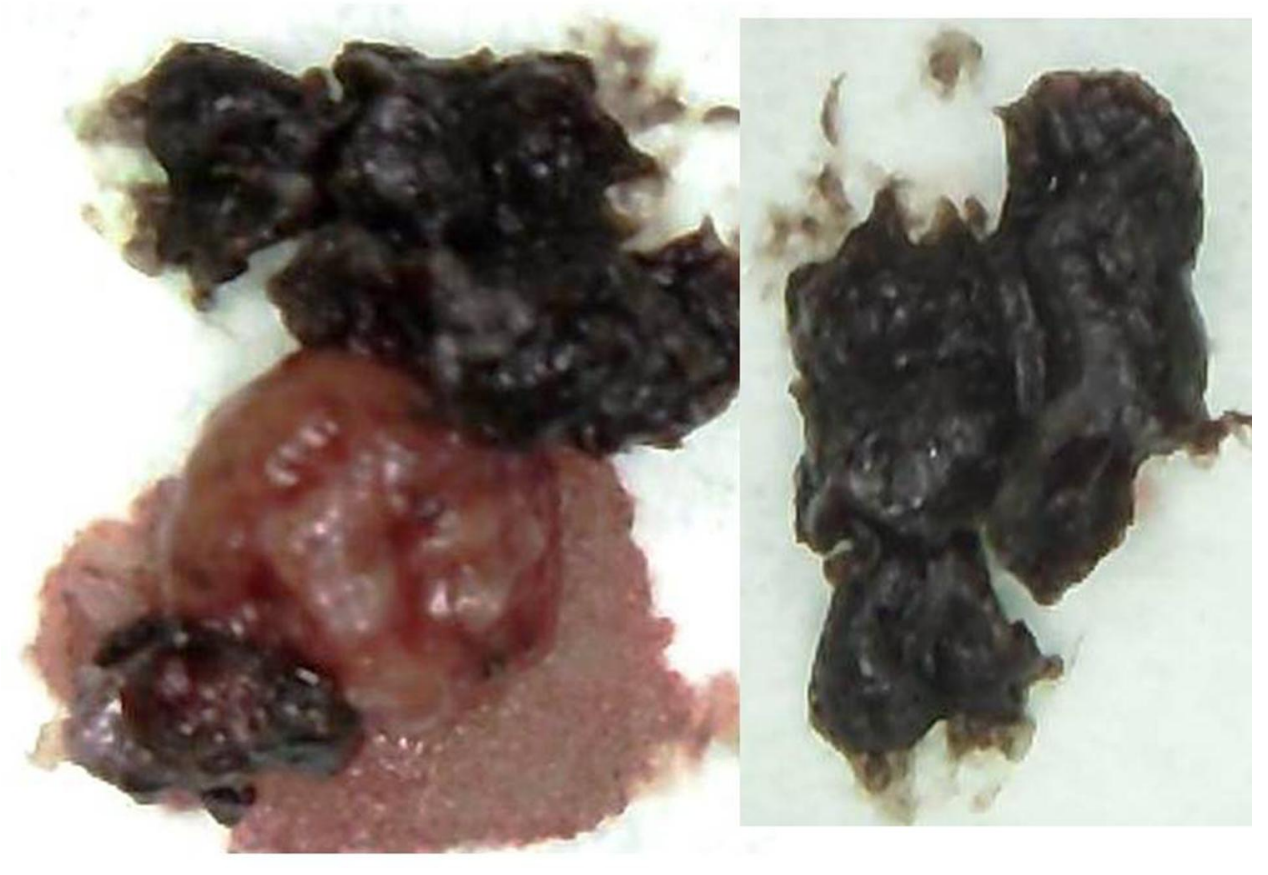

Figure 5 Aspergilloma removed from the cancellous medullary cavity in edentulous jaw area 38/39.

Note: Within the cancellous medullary cavity in the edentulous jaw area $38 / 39$, an aspergilloma ("fungal ball") formed a large, spherical colony of mold together with a fungal network that also contained a mixture of inflammatory cells (see the "Histological Findings" section).

\section{RANTES/CCL5 (pg/ml)}

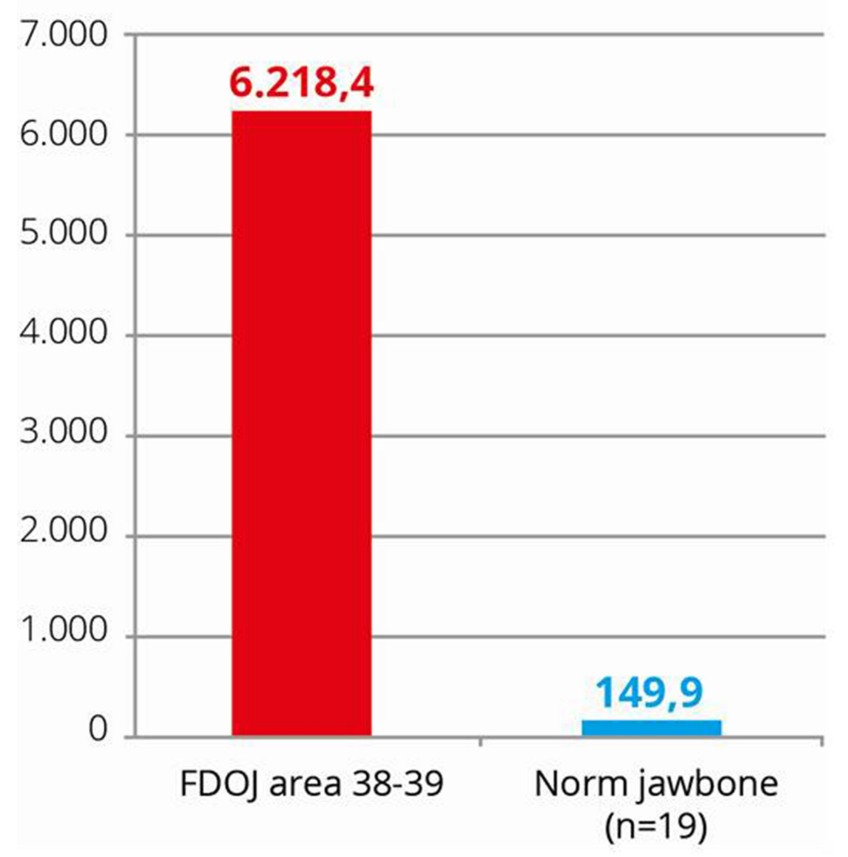

Figure 6 RANTES/CCL5 expression levels in BMDJ/FDOJ and normal jawbone areas.

Notes: The red column corresponds to the local RANTES/CCL5 expression (in pg/ $\mathrm{mL}$ ) found in BMDJ/FDOJ area 38/39. The blue column corresponds to the RANTES/CCL5 expression (in $\mathrm{pg} / \mathrm{mL}$ ) found in healthy cancellous bone.

Abbreviations: BMDJ, bone marrow defect in jawbone; FDOJ, fatty degenerative osteonecrosis of the jawbone. in $\mathrm{BMDJ} / \mathrm{FDOJ},{ }^{13-15}$ including in the specific case presented here. ${ }^{16}$ The striking result arising from the multiplex analysis of the BMDJ/FDOJ area indicated the marked elevation of the chemokine R/C.

Since there is little known about the direct clinical relationship between $\mathrm{R} / \mathrm{C}$ signaling pathways detected in BMDJ/FDOJ and an organic-specific or systemic disease, the increased $\mathrm{R} / \mathrm{C}$ level found in BMDJ/FDOJ may provide insights into possible causal pathways. ${ }^{14,17}$ As in this case of CFS, for which the etiology is unclear, the possibility of BMDJ/FDOJ involvement was raised even though the patient did not present with obvious signs or symptoms, as numerous scientific publications demonstrate a relationship between $\mathrm{R} / \mathrm{C}$ and central nervous system disorders. ${ }^{18,19}$

\section{Fungal Toxins}

This case of morphologically and histologically confirmed aspergilloma that was found broadens the discussion concerning causal pathways to include fungal toxins and their relationship to cases of CFS. Aspergillus is known to produce toxic compounds called mycotoxins, primarily aflatoxin and gliotoxins. These mycotoxins, which are formed by pathogenic Aspergillus, may contribute to the development of potentially serious and difficult-to-detect 


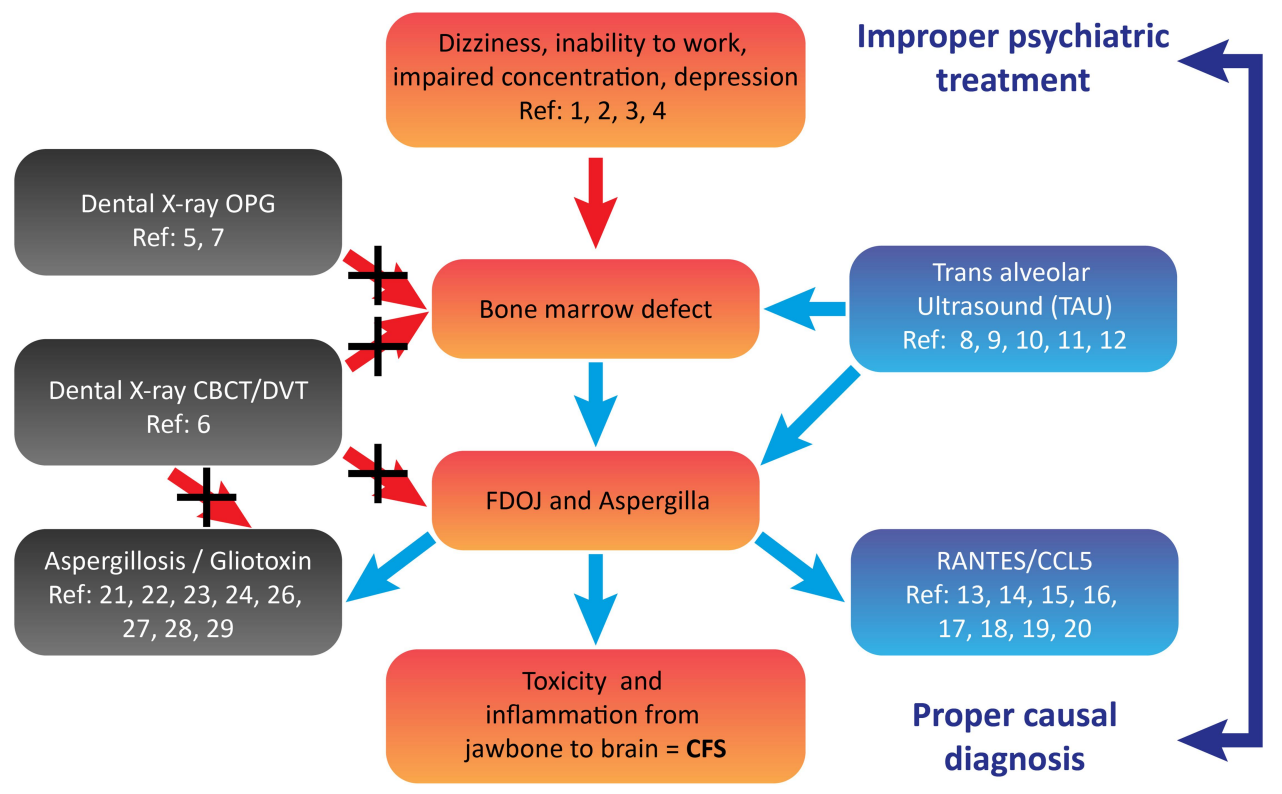

Figure 7 Case report overview including corresponding references.

Notes: Top: at the top of the graph are the disorders of the patient's state of mind that have so far been treated by unsuccessful psychotherapy. Left row: both OPG and CBCT/DVT do not lead to the detection of the bone marrow defect (center) and the FDOJ area (center, red arrows). In contrast, additional TAU examination leads to the detection of these bone marrow defects (right row, blue arrows). Postoperatively, the chronically overexpressed proinflammatory RANTES/CCL5 signaling pathways and aspergillosis can now be diagnosed in the bone marrow defects (blue arrows). Bottom: for the patient's CFS, this enables aproper causal diagnosis via the link of toxicity and inflammation from jawbone to brain.

Abbreviations: OPG, orthopantomogram; TAU, trans-alveolar ultrasound; DVT, digital volume tomogram; CBCT, cone beam computed tomography; FDOJ, fatty degenerative osteonecrosis of the jaw; CFS, chronic fatigue syndrome.

diseases. Aflatoxins have acute hepatotoxic effects at concentrations of $\sim 10 \mu \mathrm{g} / \mathrm{kg}$ of body weight, but are carcinogenic at even lower concentrations and particularly when exposed to repeatedly. ${ }^{20,21}$ In the case described, had the aspergillosis not been eliminated, the fungal toxins may have penetrated further into other tissues, involving one or more organs beyond the affected area of the jawbone.

Gliotoxin is a toxic agent that is produced by pathogenic yeasts and fungi. This compound is an active sulfur reagent of fat-soluble thiol alcohol and is an antiphagocytic and immunomodulating substance. ${ }^{22,23}$ The structure of gliotoxin is decisive for toxicological analysis: gliotoxin reacts with a sulfhydryl group on active enzyme proteins and deactivates the enzyme. This also applies to the mitochondrial enzymes in the respiratory chain. ${ }^{24,25}$ Due to this affinity for binding, there are intensive biochemical and medically relevant interactions of gliotoxin: Gliotoxin suppresses the immune system, ${ }^{26}$ thereby causing DNA damage. ${ }^{27}$ Gliotoxin also causes oxidative damage to the plasmid and cellular DNA. ${ }^{28}$ As an immunomodulating substance, gliotoxin causes genomic DNA fragmentation. ${ }^{28}$

\section{Summary}

In this case report, the cause of the patient's systemic symptoms appears to be caused by possible irritations of the central nervous system due to $\mathrm{R} / \mathrm{C}$ overexpression. ${ }^{16,18,19}$ The problem associated with BMDJ/FDOJ begins when local, acute inflammation present in the jaw at the initial stage of the wound healing process transitions to a sub-chronic stage. The osteolytic BMDJ/FDOJ area in association with chemokine $\mathrm{R} / \mathrm{C}$ overexpression may induce numerous unspecific symptoms and diseases. ${ }^{18,19}$ At the same time, the mycotoxins formed in the medullary cavity of the jawbone may strongly contribute to the onset and maintenance of a chronic inflammatory condition that may favor the CFS (Figure 7). The histologically confirmed findings in area 38 and retromolar area of the patient's jaw indicate that the innate capacity for wound healing, without prior surgical debridement, was significantly impaired, which not only lead to an $\mathrm{R} / \mathrm{C}$ overexpression in this case but also, in this case to a highly toxic fungal contamination. 


\section{Conclusion}

The clinical examination and radiographic imaging performed by our team demonstrated that, X-ray diagnostics alone would not have identified a potential contributing cause of the patient's health condition. Neither OPG nor DVT/CBCT detected any lesions that would have provided a medical indication that necessitated oral surgical intervention. It is only with the additional TAU diagnostic methods that we were able to verify the previously undiscovered phenomenon of BMDJ/FDOJ preoperatively by imaging and postoperatively by histopathological investigations. Without complementary TAU measurement indicating possible medullary osteolysis in the distal and retromolar jaw (area 38/39), neither the chronic inflammatory signaling pathways (as identified via $\mathrm{R} / \mathrm{C}$ expression) nor the extensive fungal colonization found in this area of the patient's jaw would have been recognized or eliminated. It is recommended that the results presented herein are used to guide further research, in particular, the demonstrated measuring of inflammatory cytokines found in bone tissue and visualize diminished bone density in bone marrow defects by TAU. The limitation of TAU lies in not displaying a detailed picture of bone structures but of only measuring bone density. Our findings in this case report of chronic fatigue syndrome clearly demonstrates the strong need of further examinations of BMDJ/FDOJ.

\section{Acknowledgments}

English-language editing of this manuscript was provided by Natasha Gabriel.

\section{Disclosure}

CaviTAU $^{\circledR}$ (Munich, Germany), the company that designed the new TAU apparatus and associated software, provided this equipment without charge for the purposes of this report. The ultrasonography procedure was carried out at the Clinic for Integrative Dentistry (Munich, Germany). CaviTAU ${ }^{\circledR}$ and the Clinic for Integrative Dentistry are engaged in ongoing discussions to further improve and verify the new TAU apparatus, CaviTAU ${ }^{\circledR}$, as it is introduced to the market. The author is the holder of a patent PTC/EP2018/084199 used in the CaviTAU ${ }^{\circledR}$. The authors report no other conflicts of interest in this work.

\section{References}

1. Hawkes N. Better training is needed to deal with increasing multimorbidity. BMJ. 2012;344:e3336. doi:10.1136/bmj.e3336
2. Barnett K, Mercer SW, Norbury M, Watt G, Wyke S, Guthrie B. Epidemiology of multimorbidity and implications for health care, research, and medical education: a cross-sectional report. Lancet. 2012;380(9836):37-43. doi:10.1016/S0140-6736(12)60240-2

3. Smith SM, Soubhi H, Fortin M, Hudon C, O'Dowd T. Interventions for improving outcomes in patients with multimorbidity in primary care and community settings. Cochrane Database Syst Rev. 2012;4: CD006560.

4. Jakovljevic M, Reiner Z, Milicic D, Crncevic Z. Comorbidity, multimorbidity and personalized psychosomatic medicine: epigenetics rolling on the horizon. Psychiatr Danub. 2010;22(2):184-189.

5. Lechner J, Schuett S, von Baehr V. Aseptic-avascular osteonecrosis: local "silent inflammation" in the jawbone and RANTES/CCL5 overexpression. Clin Cosmet Investig Dent. 2017;9:99-109. doi:10.2147/ CCIDE.S149545

6. Guerrero M, Jacobs R, Loubele M, Schutyser F, Suetens P, van Steenberghe D. State-of-the-art on cone-beam CT imaging for preoperative planning of implant placement. Clin Oral Investig. 2006;10:1-7. doi:10.1007/s00784-005-0031-2

7. Lechner J. Validation of dental X-ray by cytokine RANTES - comparison of X-ray findings with cytokine overexpression in jawbone. Clin Cosmet Investig Dent. 2014;6:71-79. doi:10.2147/CCIDE.S69807

8. Bouquot J, Martin W, Wrobleski G. Computer-based thru-transmission sonography (CTS) imaging of ischemic osteonecrosis of the jaws - a preliminary investigation of 6 cadaver jaws and 15 pain patients. Oral Surg Oral Med Oral Pathol Oral Radiol Endod. 2001;92:550.

9. Al-Nawas B, Grotz KA, Kann P. Ultrasound transmission velocity of the irradiated jaw bone in vivo. Clin Oral Invest. 2001;5(4):266-268. doi:10.1007/s00784-001-0133-4

10. Klein MO, Grotz KA, Manefeld B, Kann PH, Al-Nawas B. Ultrasound transmission velocity for non-invasive evaluation of jaw bone quality in vivo prior to dental implantation. Ultrasound Med Biol. 2008;34:1966-1971. doi:10.1016/j.ultrasmedbio.2008.04.016

11. Lechner J, Zimmermann B, Schmidt M, von Baehr V. Ultrasound sonography to detect focal osteoporotic jawbone marrow defects: clinical comparative report with corresponding Hounsfield units and RANTES/CCL5 expression. Clin Cosmet Investig Dent. 2020;12:205-216. doi:10.2147/CCIDE.S247345

12. Lechner J, von Baehr V, Zimmermann B. Osteonecrosis of the jaw beyond bisphosphonates: are there any unknown local risk factors? Clin Cosmet Investig Dent. 2021;13:21-37. doi:10.2147/CCIDE.S288603

13. Lechner J, Mayer W. Immune messengers in neuralgia inducing cavitational osteonecrosis (NICO) in jaw bone and systemic interference. Eur J of Integrative Med. 2010;2:71-77. doi:10.1016/j. eujim.2010.03.004

14. Lechner J, von Baehr V. RANTES and fibroblast growth factor 2 in jawbone cavitations: triggers for systemic disease? Int J Gen Med. 2013;6:277-290. doi:10.2147/IJGM.S43852

15. Lechner J, von Baehr V. Hyperactivated signaling pathways of chemokine RANTES/CCL5 in osteopathies of jawbone in breast cancer patients-case report and research. Breast Cancer (Auckl). 2014;8:89-96. doi:10.4137/BCBCR.S15119

16. Lechner J, Huesker K, von Baehr V. Impact of RANTES from jawbone on chronic fatigue syndrome. $J$ Biol Regul Homeost Agents. 2017;31(2):321-327.

17. Lechner J, von Baehr V. Chemokine RANTES/CCL5 as an unknown link between wound healing in the jawbone and systemic disease: is prediction and tailored treatments in the horizon? EPMA J. 2015;6 (1):10. doi:10.1186/s13167-015-0032-4

18. Bolin LM, Murray R, Lukacs NW, et al. Primary sensory neurons migrate in response to the chemokine RANTES. $J$ Neuroimmunol. 1998;81(1-2):49-57. doi:10.1016/S0165-5728(97)00158-6

19. Luo Y, Berman MA, Zhai Q, et al. RANTES stimulates inflammatory cascades and receptor modulation in murine astrocytes. Glia. 2002;39 (1):19-30. doi:10.1002/glia.10079 
20. Frisvad JC, Thrane U, Samson RA, Pitt JI. Important mycotoxins and the fungi which produce them. Adv Exp Med Biol. 2006;571:3-31.

21. Yamada A, Kataoka T, Nagai K. The fungal metabolite gliotoxin: immunosuppressive activity on CTL-mediated cytotoxicity. Immunol Lett. 2000;71(1):27-32. doi:10.1016/S0165-2478(99)00155-8

22. Shah DT, Jackman S, Engle J, Larsen B. Effect of gliotoxin on human polymorphonuclear neutrophils. Infect Dis Obstet Gynecol. 1998;6(4):168-175. doi:10.1155/S1064744998000349

23. Sutton P, Warig P, Mullbacher A. Exacerbation of invasive aspergillosis by the immunosuppressive fungal metabolite, gliotoxin Immunol Cell Biol. 1996;74(4):318-322. doi:10.1038/icb.1996.57

24. Sutton P, Newcombe NR, Waring P, Mullbacher A. In vivo immunosuppressive activity of gliotoxin, a metabolite produced by human pathogenic fungi. Infect Immun. 1994;62(4):1192-1198. doi:10.1128/ IAI.62.4.1192-1198.1994
25. Eichner RD, Al Salami M, Wood PR, Mullbacher A. The effect of gliotoxin upon macrophage function. Int J Immunopharmacol. 1986;8(7):789-797. doi:10.1016/0192-0561(86)90016-0

26. Mullbacher A, Eichner RD. Immunosuppression in vitro by a metabolite of a human pathogenic fungus. Proc Natl Acad Sci U S A. 1984;81(12):3835-3837. doi:10.1073/pnas.81.12.3835

27. Wang JS, Groopman JD. DNA damage by mycotoxins. Mutat Res. 1999;424(1-2):167-181. doi:10.1016/S0027-5107(99)00017-2

28. Zhou X, Zhao A, Going G, Hirszel P. Gliotoxin-induced cytotoxicity proceeds via apoptosis and is mediated by caspases and reactive oxygen species in LLC-PK1 cells. Toxicol Sci. 2000;54(1):194-202. doi:10.1093/toxsci/54.1.194

\section{Publish your work in this journal}

The International Medical Case Reports Journal is an international, peer-reviewed open-access journal publishing original case reports from all medical specialties. Previously unpublished medical posters are also accepted relating to any area of clinical or preclinica science. Submissions should not normally exceed 2,000 words or 4 published pages including figures, diagrams and references. The manuscript management system is completely online and includes a very quick and fair peer-review system, which is all easy to use. Visit http://www.dovepress.com/testimonials.php to read real quotes from published authors. 